\title{
The role of nutrition and dietary supplements in the management of diarrhoea in HIV patients: a review of the literature
}

\author{
Ivan Armando Osuna-Padilla ${ }^{1,2}$, Claudia Patricia Maza-Moscoso³, Isabel Martínez Del Río-Requejo ${ }^{4}$ \\ ${ }^{1}$ Centro de Investigación en Enfermedades Infecciosas, Instituto Nacional de Enfermedades Respiratorias, Mexico City, Mexico \\ ${ }^{2}$ Sección de Estudios de Posgrado e Investigación, Escuela Superior de Medicina, Instituto Politécnico Nacional, Mexico City, Mexico \\ ${ }^{3}$ Department of Nutrition and Dietetic, Centro Médico Militar, Guatemala City, Guatemala \\ ${ }^{4}$ Nutrition Division, Hospital 20 de Noviembre, ISSSTE, Mexico City, Mexico
}

\begin{abstract}
Diarrhoea is present in $28-60 \%$ of people living with human immunodeficiency virus (HIV). This condition is associated with malabsorption, nutritional risk, and higher incidence of infections and is one of the main causes of morbidity and mortality. Diarrhoea may be classified by duration and aetiology. Acute and chronic diarrhoea causes a reduction in dietary intake and also reduces the ability to absorb nutrients, leading to patient malnutrition and impacting their quality of life. The medical treatment for infectious diarrhoea is the eradication of pathogens; however, there is no specific treatment for non-infectious diarrhoea, which suggests the use of antisecretory medications, antimotility, and adsorbent agents. The use of non-pharmacological strategies such as the dietary modifications and nutrition supplements, specifically zinc, elemental diets, glutamine, fibre, and probiotics may decrease the duration of diarrhoea and may ameliorate the frequency and consistency of stool depositions. The goal of nutritional intervention is to promote the intake of energy and protein amounts needed to maintain or improve the nutritional status and quality of life. Despite promising data in individual strategies, further studies are needed to evaluate the multiple approaches in clinical practice. In this review, we aim to summarise the nutritional management and supplementation strategies for diarrhoea in HIV patients.
\end{abstract}

HIV AIDS Rev 2019; 18, 3: 158-164 DOI: https://doi.org/10.5114/hivar.2019.88270

Key words: diarrhoea, HIV, nutrition, immunonutrition, probiotic, fibre.

\section{Introduction}

Although progress has been made in the global fight against human immunodeficiency virus (HIV), the prevalence continued to increase and diarrhoea is one of the main causes of morbidity and mortality, being present in $28-60 \%$ of people living with HIV (PLWHIV) [1,2]. HIV infection per se leads to alterations in the intestinal barrier integrity with changes in the following: (a) the biological barrier, producing a variation in the composition of the intestinal microbiota, a reduction in Bifidobacterium and lactobacilli, and an increase in opportunistic pathogen families, such as Pseudomonas aeruginosa and Candida albicans; (b) the immunological barrier, with a massive depletion of CD4+ and Th17 cells in the intestine, altering the balance of immunological cells; and (c) the mechanical barrier, with atrophy in the intestinal villi and alterations in the expression of intestinal tight junctions [3].

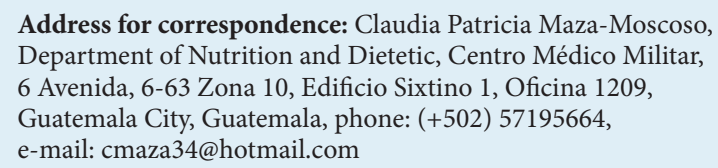

Address for correspondence: Claudia Patricia Maza-Moscoso, Department of Nutrition and Dietetic, Centro Médico Militar, 6 Avenida, 6-63 Zona 10, Edificio Sixtino 1, Oficina 1209, Guatemala City, Guatemala, phone: (+502) 57195664, e-mail:cmaza34@hotmail.com

\section{Article history:}

Received: 28.02.2019

Received in revised form: 05.06.2019

Accepted: 07.06.2019

Available online: 09.09.2019
International Journal of HIV-Related Problems

HIV \& AIDS

R e vi e w 


\section{Definition and classification of diarrhoea}

The World Health Organization define diarrhoea as "the passage of three or more loose or liquid stools per day, or more frequently than normal for the individual" [4]. The Bristol Stool Chart scale may be useful to evaluate the consistency of evacuations, where types 5, 6, and 7 tend toward diarrhoea [5].

Diarrhoea may be classified by duration and aetiology. Considering the duration, diarrhoea is classified as acute when the duration is from 0 to 13 days, persistent when existing from 14 to 29 days, and chronic when the duration is more than 30 days $[6,7]$.

Considering the aetiology, diarrhoea is classified as infectious or non-infectious. Infectious diarrhoea is common in HIV patients who are not receiving antiretroviral therapy (ART) because intestinal alterations lead to bacterial translocation, defined by Zevin et al. as the movement of microbial products from the gut mucosa into circulation [8], which are phagocytosed and contribute to the intestinal inflammatory response and predispose to developing diarrhoea by overgrowth of opportunistic bacterial, protozoal, viral, and fungal pathogens in the intestinal lumen [1]. On the other hand, some translocated bacteria and toxic compounds are drained by the mesenteric lymph system, which can induce the development of chronical local and systemic immune stimulation [8].

The most common microorganisms that cause diarrhoea in PLWHIV are: Escherichia coli, Cryptosporidium, Microsporidia, Cystoisospora belli, Cytomegalovirus, and Mycobacterium avium complex [7].

Non-infectious diarrhoea in PLWHIV is caused by alterations in the mechanical barrier, also known as enteropathy associated with HIV or associated with ART [9], which is defined as an idiopathic form of diarrhoea where no pathogen agent is identified, and it is characterised by histological changes in the intestinal microarchitecture. Malabsorption is a consequence of this type of diarrhoea, which leads to weight loss and malnutrition [10]. Diarrhoea is also an adverse effect of ART, mainly in those treated with protease inhibitors drugs, which increase the water and electrolyte secretion to the intestinal lumen, although all medicines generally cause a higher incidence of diarrhoea [11].

\section{Nutritional implications of diarrhoea in HIV}

Acute and chronic diarrhoea have serious implications for the nutritional status of PLWHIV, reducing food intake and the ability to nutrient absorption, which leads to malnutrition. An observational study of faecal losses of energy (measured by bomb calorimetry) in critically ill patients with severe diarrhoea showed a low intestinal energyabsorption capacity in patients who evacuated more than $350 \mathrm{~g} /$ day, and documented a faecal energy loss of 445.5 $\pm 201.3 \mathrm{kcal} /$ day [12].
Chronic diarrhoea has other nutritional implications including fat and muscle wasting and higher prevalence of nutritional deficiencies, mainly vitamin $\mathrm{B}_{12}$. However, no prevalence data are available [13]. Additionally, infectious diarrhoea is associated with increased catabolism, higher use of nutrients, reduction in quality of life [14], and increased risk of infection due to the reduction of immune response and damage to the intestinal mucosa [15].

\section{Nutritional management}

In infectious diarrhoea, the medical treatment is the eradication of identified pathogens; however, there is no specific treatment for non-infectious diarrhoea. The use of antisecretory medications, antimotility agents, adsorbent agents, and others is suggested [16].

The use of non-pharmacological strategies, including dietary modifications and supplementation of some nutrients, could have an impact on the number and consistency of stool depositions; however, there are a lack of clinical trials evaluating the effectiveness of nutrition therapy [17]. Recommendations from guidelines and literature reviews remark the importance to obtain a food intake history from each patient with the aim of quantifying food and nutrient consumption, in order to establish the association of food intake and symptoms and assess the adequacy of intake. Capili et al. analysed nutrient intake (seven-day food diary) in a sample of 75 PLWHIV on ART with non-pathogen diarrhoea, and reports suboptimal intake of fibre due to the low consumption of fruit and vegetables [18, 19].

\section{Dietary modifications}

The aim of nutritional intervention is to promote the intake of optimal amounts of energy and protein in order to maintain or improve the patient's nutritional status and quality of life. Resting energy expenditure should be measured by indirect calorimetry or estimated using Mifflin St. Jeor equations in patients with normal weight and HarrisBenedict in obese patients [20]. It is important to take into account the aetiology of diarrhoea to design the best nutritional strategy, and to consider the provision of a higher energy supply (50-100\% extra) in cases of malabsorption. The Academy of Nutrition and Dietetics recommends $1.0-1.4 \mathrm{~g} / \mathrm{kg}$ of protein for maintenance of the body mass or $1.5-2.0 \mathrm{~g} / \mathrm{kg}$ in malnutrition status [21]. Other international societies suggest an energy prescription considering the HIV classification proposed by the Centres for Disease Control and Prevention, providing $30-35 \mathrm{kcal} / \mathrm{kg}$ in patients in phase $\mathrm{A}, 35-40 \mathrm{kcal} / \mathrm{kg}$ in phase $\mathrm{B}$, and $40-50 \mathrm{kcal} / \mathrm{kg}$ in phase C; however, there are no clinical data about the accuracy of this method [22].

Clinical guidelines state that certain foods may aggravate or reduce chronic diarrhoea [17] because the damage to the intestinal mucosa reduces the absorption of carbohydrates which increases in the intestinal lumen, causing 
Table 1. Dietary recommendations for patients with acute and chronic diarrhoea

\begin{tabular}{l} 
Drink the appropriate amount of liquids to avoid \\
dehydration. \\
\hline Drink rehydrating solutions either with ready to use \\
products or home preparations. The latter may be prepared \\
mixing a spoon of salt $(3 \mathrm{~g})$ and 8 spoons of sugar ( $18 \mathrm{~g})$ \\
per litre of potable water. \\
\hline Increase the frequency and reduce the amount of food. \\
\hline Eat foods rich in soluble fibre. \\
\hline Eat starched foods such as rice, corn, potatoes, and pasta. \\
\hline Eat foods rich in proteins and low in fats, such as eggs, \\
chicken, or fish. \\
\hline Avoid fried preparations and fructose food source.
\end{tabular}

liquid retention due to osmolality and fermentation by gut microbiota, increasing gastrointestinal symptoms. Taking this into consideration, restriction of refined sugars and lactose is recommended, attention has been focused on reducing the supply of foods rich in fructose (e.g. fruit juices and other sweet beverages). On the other hand, high-fat diets are associated with a loss of liquids, electrolytes, bile acids, and divalent cations to the intestinal lumen; therefore it is recommended that the amount of fat in the diet is reduced; caffeine restriction is also recommended due to the stimulation peristaltic movement. Lack of clinical trials evaluating the impact of food restriction in HIV-positive patients are available. Anastasi et al. evaluated in 65 patients with PLWHIV the impact of dietary modifications (diet low in fat, lactose, and insoluble fibre) and reported a reduction of $28 \%$ in the frequency of stool depositions and improvement in faecal consistency after six months of intervention [23].

Eat small meals recommendation (more than three meals per day) may be useful to increase the consumption of nutrients and promote longer use of the absorption surface area in the intestine for more time during the day [18]. There is a lack of evidence to support this recommendation alone, but these interventions may be useful as part of a comprehensive intervention plan.

FODMAP is an acronym that stands for FermentableOligo-Di-Monosaccharides and Polyols. These are carbohydrates that are present naturally in many foods and play an important role in inducing diarrhoea and other symptoms in irritable bowel syndrome (IBS) patients; fructose and polyols are absorbed slowly in the small intestine; lactose cannot be digested by many patients; and oligosaccharides also cannot be digested. After intake of a meal high in FODMAP or gluten, these carbohydrates pass undigested into the large intestine and are fermented, producing gas, diarrhoea, bloating and distention in the IBS population [24]. Mechanisms of diarrhoea in IBS patients are different from those of HIV+ patients; however, bacterial translocations are present in both conditions (intestinal permeability cor- relates with stool frequency in IBS conditions [25]), and one cross-sectional study reports a greater prevalence of IBS in HIV-positive compared with HIV-negative subjects (10.2\% vs. $2.2 \%, p \leq 0.002$ ) [26], which might be explained by the possible role of some pathogenic infections in the development and exacerbation of this condition [27]. Some studies suggest that a low-FODMAP or gluten-free diet might improve stool consistency and frequency in IBS $[24,28,29]$ by improving intestinal permeability, but no evidence from observational or clinical trial data in HIV patients are available.

In patients with suboptimal energy and protein intake, prescription of elemental nutrition formulations (containing hydrolyzed whey protein and medium-chain triglycerides) may be considered, in order to increase the intake of nutrients and improve their absorption [30]. Salomon et al. reported a decrease in the number of stools and improvement in faecal fat excretion after nine days of enteral feeding with an elemental diet in 11 PLWHIV and diarrhoea [31]. In a randomised trial comparing elemental formula (85\% of medium-chain triglycerides, $n=13$ ) and control formula $(100 \%$ long-chain triglycerides, $n=10)$ in patients with malabsorption and HIV, Craig et al. reported a decreased in stool fat and stool nitrogen, an increase in fat absorption, and a trend toward as decreased number of bowel movements after 12 days of elemental formula [32]. Limitations of both studies were the number of individuals included in the analysis and the limited data about the randomisation process.

Vitamin and mineral supplementation must be considered in patients with suboptimal intake, mainly in cases of malabsorption. Total parenteral nutrition (TPN) prescription does not provide greater benefit than enteral nutrition or oral nutritional supplements and is more expensive [33, 34]; however, it may be considered in patients who are nutritionally at-risk or malnourished, and who unlikely to achieve energy and protein goals in the first three to five days, according the American Society for Parenteral and Enteral Nutrition (ASPEN) [35].

It is important to consider that once the pharmacological therapy is initiated in patients with infectious diarrhoea, improvement is expected in the short-term. In these cases, the purpose of the nutritional management is to maintain adequate hydration and optimal intake of nutrients and electrolytes [36]. Some dietetic recommendations are mentioned in Table 1.

\section{Nutritional supplementation strategies}

\section{Zinc}

Zinc deficiency has negative effects on the immune system and exacerbates diarrhoea by diverse mechanisms: a) on innate response, deficiency reduces the phagocytic capacity of macrophages and neutrophils as well as NK activity, increasing the vulnerability to infections, b) on adaptive response, it reduces lymphocytes count and proliferation 
mediated by thymus apoptosis and atrophy. In the gut barrier, zinc deficiency alters liquid secretion [37].

For diarrhoea treatment, World Health Organization suggests the supplementation of $10 \mathrm{mg}$ /day in children $<6$ months old for 10-14 days, and $20 \mathrm{mg}$ /day in those $>6$ months old [38]. A systematic review of 33 randomised clinical trials in children $>6$ months old showed that zinc supplementation can reduce the duration of diarrhoea (MD $=-11.46$ hours, 95\% CI: -19.72 to -3.19 hours). In children with malnutrition, the reduction of diarrhoea seems to be more significant ( $\mathrm{MD}=-26.39$ hours, $95 \% \mathrm{CI}:-36.54$ to -16.23 hours) [39].

There is no established dose for supplementation in HIV adult patients. Cárcamo et al. supplemented $100 \mathrm{mg} /$ day of elemental zinc in patients with persistent diarrhoea and did not observe any improvement in diarrhoea after 14 days of supplementation [40]. Baum et al. observed an improvement in the incidence of diarrhoea (OR $=0.40,95 \% \mathrm{CI}$ : 0.183 to $0.981, p=0.019$ ) after supplementation of $12 \mathrm{mg}$ in women and $15 \mathrm{mg}$ in men for 18 months in a sample of 231 PLWHIV with low plasma zinc levels [41]. Among other benefits in PLWHIV, zinc supplementation has shown an increased CD4+ count, improved serum concentration of zinc, and prevention of opportunistic infections [42].

\section{Glutamine}

Glutamine represents the most abundant amino acid present in plasma, which plays an important role in various metabolic and biochemical processes. One of them is the regulation of the integrity in the intestinal surface by increasing protein synthesis in the epithelial intestinal cells [43].

Different authors have evaluated the safety and efficacy of glutamine supplementation in PLWHIV. Noyer et al. documented improvements in the intestinal permeability after glutamine supplementation for 28 days in patients with intestinal permeability diagnosed by alterations in the mannitol and lactulose test, using an oral dose of glutamine of 4-8 g/day, suggesting doses of at least $20 \mathrm{~g} /$ day [44]. Similar results were reported by Bushen et al. after supplementing oral doses of $30 \mathrm{~g} /$ day of glutamine or $44 \mathrm{~g} /$ day of alanine glutamine dipeptide for seven days in PLWHIV [45]. Subsequently, Huffman et al. documented a reduction in the severity of diarrhoea associated with ART (nelfinavir), as well as an improvement in the quality of life, using an oral dose of $30 \mathrm{~g} /$ day L-glutamine for 10 days in 25 PLWHIV [46]. Recently, Leite et al. evaluated the effect of glutamine in the intestinal permeability in 46 PLWHIV, and reported an increase in the excreted urinary mannitol, after 10 days of an oral solution that contain $24 \mathrm{~g}$ of alanine glutamine dipeptide [47]. More recently, an in vitro study in colon biopsies of IBS patients reported an increase in the expression of tight junctions, specifically in Claudin-1, after exposure to different glutamine concentrations, which could explain such benefits in the intestinal permeability improvement and the diarrhoea episodes [48]. Currently there is no consensus about the dose to be used in PLWHIV. Therefore, the recommendations of ASPEN could be followed, suggesting a dose of $0.2-0.5 \mathrm{~g} / \mathrm{kg} /$ day [49].

\section{Fibre and prebiotics}

Fibre is defined as polymers of carbohydrates, which are not hydrolysed by enzymes in the gut, while prebiotics are non-digestible compounds that, after being metabolised by the microorganisms in the intestine, have modulatory functions in the composition and activity of the gut microbiota, producing benefits to the host. Both components have been studied due to their possible benefit in PLWHIV with diarrhoea, specifically in diarrhoea mediated by microbial toxins that alter fluid balance and electrolytes in the intestinal lumen. The use of prebiotics (fructooligosaccharides, oligosaccharides, and inulin) has been suggested as a strategy to prevent diarrhoea and gel forming fibres (Plantago psyllium and guar gum) [50]. ASPEN suggests the use of fermentable soluble fibres (fructooligosaccharides and inulin); specifically, 10-20 g divided into several doses during a period of 24 hours in patients with severe diarrhoea [51], although there is a lack of evidence to support this recommendation. Recently, a meta-analysis that included three randomised clinical trials of two prebiotics and one product of prebiotic fermentation (fructooligosaccharides, galactooligosaccharide, and sodium butyrate) did not show and effect on diarrhoea prevention ( $\mathrm{RR}=0.83,95 \mathrm{CI} \%$ : 0.58-1.18) [52].

There are no guidelines about the dosage and type of fibres to be used in HIV patients; however, some cross-sectional studies have shown that patients who take Plantago psyllium have reduced incidence of diarrhoea and better consistency in their faeces; therefore, it should be considered as a low-cost strategy [53]. The action mechanism of P. psyllium is its capacity to form gels, contributing to the retention of liquids present in the intestinal lumen, thus improving the stool consistency and frequency of evacuations [54]. Some authors suggest a dose of 18-30 g/day of P. psyllium to normalise the stools in patients with chronic diarrhoea, suggesting a gradually increasing dose to improve tolerance [55]. More recently, Lertpipopmetha et al. reported no beneficial effect on reducing incidence of diarrhoea in general medical patients ( $43 \%$ with infectious diseases) receiving enteral nutrition enriched with $15.2 \mathrm{~g} / \mathrm{l}$ of psyllium fibre [56].

\section{Probiotics}

Probiotics are "live microorganisms which when administered in adequate amounts confer a health benefit on the host" [57]. Several meta-analyses have shown a possible benefit after their use in severe infectious diarrhea and in persistent diarrhea in children and adult patients $[58,59]$.

Probiotics can reduce infectious diarrhoea in PLWHIV through several mechanisms: (a) they can compete for nutrients and adhere to the epithelium and intestinal mucosa, 
inhibiting the epithelial invasion by pathogens and preventing the bacterial translocation; and (b) they stimulate the production of antimicrobial substances and $\operatorname{IgA}[60]$. However, their effectiveness is not clear because a meta-analysis published by Carter et al., which included nine clinical trials, did not find benefits in the management of diarrhoea associated with HIV, documenting a possible benefit when products with Saccharomyces boulardii were used [61].

A randomised clinical trial conducted by Santos et al. evaluated the effectiveness of two treatments for reduction of gastrointestinal symptoms in adult patients with ART: group $1(n=25)$ received nutritional treatment + maltodextrin and group $2(n=23)$ received nutritional treatment + symbiotic (6 g fructooligosaccharides and Lactobacillus rhamnosus, L. paracasei, L. acidophilus and Bifidobacterium lactis) for six months. Significant reduction in diarrhoea incidence in both groups was reported, but no difference between the groups was observed [62].

Bacteraemia caused by the use of probiotics is reported in the literature; therefore, their use should not be widespread [63, 64]. The Academy of Nutrition and Dietetics (AND) suggests monitoring the consumption of probiotics in patients with counts of CD4+ $<200$ cells $/ \mathrm{mm}^{3}$ and in those with $\mathrm{CD} 4+<15 \%$ [21].

The use of probiotics for the treatment of non-infectious diarrhoea in PLWHIV, especially those receiving ART, is not supported by literature. There is some controversy about probiotics causing damage in patients receiving ART with immunological reconstitution [11].

\section{Conclusions}

HIV infection causes anatomic, structural, and biological alterations to the gut, resulting in a greater incidence of diarrhoea episodes. Infectious diarrhoea is common in HIV patients who are not receiving ART, due to intestinal alterations caused by bacterial translocation. Non-infectious diarrhoea is usually present due to enteropathy associated with HIV and as a secondary effect of ART. Acute and chronic diarrhoea cause a reduction in dietary intake and also reduce the ability to absorb nutrients, leading to patient malnutrition and impacting the quality of life. The medical treatment for infectious diarrhoea is the eradication of pathogens; however, there is no specific treatment for non-infectious diarrhoea, which suggests the use of antisecretory medications, antimotility, and adsorbent agents, as well as the use of non-pharmacological strategies such as the dietary modifications and nutrition supplements like zinc, glutamine, fibre, and probiotics.

\section{Acknowledgments}

The authors would like to thank Mrs. Miriam Moscoso De Maza for her assistance in technical editorial and medical writing.

\section{Conflict of interest}

The authors declare no conflict of interest with respect to the research, authorship, and/or publication of this article.

\section{References}

1. Logan C, Beadsworth MBJ, Beeching NJ. HIV and diarrhoea: what is new? Curr Opin Infect Dis 2016; 29: 486-494.

2. Hall VP. Common gastrointestinal complications associated with human immunodeficiency virus/AIDS: an overview. Crit Care Nurs Clin North Am 2018; 30: 101-107.

3. Assimakopoulos SF, Dimitropoulou D, Marangos M, Gogos CA. Intestinal barrier dysfunction in HIV infection: pathophysiology, clinical implications and potential therapies. Infection 2014; 42: 951-959.

4. World Health Organization. The treatment of diarrhoea: a manual for physicians and other senior health workers. World Health Organization, Geneva 2005.

5. Reintam Blaser A, Deane AM, Fruhwald S. Diarrhoea in the critically ill. Curr Opin Crit Care 2015; 21: 142-153.

6. Riddle MS, DuPont HL, Connor BA. ACG Clinical Guideline: diagnosis, treatment, and prevention of acute diarrheal infections in adults. Am J Gastroenterol 2016; 111: 602-622.

7. Shane AL, Mody RK, Crump JA, et al. 2017 Infectious Diseases Society of America clinical practice guidelines for the diagnosis and management of infectious diarrhea. Clin Infect Dis 2017; 65: 1963-1973.

8. Zevin AS, McKinnon L, Burgener A, Klatt NR. Microbial translocation and microbiome dsybiosis in HIV-associated immune activation. Curr Opin HIV AIDS 2016; 11: 182-190.

9. Dikman AE, Schonfeld E, Srisarajivakul NC, Poles MA. Human immunodeficiency virus-associated diarrhea: still an issue in the era of antiretroviral therapy. Dig Dis Sci 2015; 60: 2236-2245.

10. Cello JP, Day LW. Idiopathic AIDS enteropathy and treatment of gastrointestinal opportunistic pathogens. Gastroenterology 2009; 136: 1952-1965.

11. Clay PG, Crutchley RD. Noninfectious diarrhea in HIV seropositive individuals: a review of prevalence rates, etiology, and management in the era of combination antiretroviral therapy. Infect Dis Ther 2014; 3: 103-122.

12. Wierdsma NJ, Peters JHC, Weijs PJM, et al. Malabsorption and nutritional balance in the ICU: fecal weight as a biomarker: a prospective observational pilot study. Crit Care 2011; 15: R264.

13. Gorospe EC, Oxentenko AS. Nutritional consequences of chronic diarrhoea. Best Pract Res Clin Gastroenterol 2012; 26: 663-675.

14. Siddiqui U, Bini EJ, Chandarana K, et al. Prevalence and impact of diarrhea on health-related quality of life in HIV-infected patients in the era of highly active antiretroviral therapy. J Clin Gastroenterol 2007; 41: 484-490.

15. Mata L. Diarrheal disease as a cause of malnutrition. Am J Trop Med Hyg 1992; 47: 16-27.

16. MacArthur RD, DuPont HL. Etiology and pharmacologic management of noninfectious diarrhea in HIV-infected individuals in the highly active antiretroviral therapy era. Clin Infect Dis Off Publ Infect Dis Soc Am 2012; 55: 860-867.

17. Li Z, Vaziri H. Treatment of chronic diarrhoea. Best Pract Res Clin Gastroenterol 2012; 26: 677-687.

18. Schiller LR. Nutrition management of chronic diarrhea and malabsorption. Nutr Clin Pract 2006; 21: 34-39.

19. Capili B, Anastasi JK. Body mass index and nutritional intake in patients with HIV and chronic diarrhea: a secondary analysis. J Am Acad Nurse Pract 2008; 20: 463-470.

20. Osuna-Padilla IA, Aguilar-Vargas A, Villazón-De la Rosa A, et al. Accuracy of predictive equations for energy expenditure in Mexicans living with HIV/AIDS with and without antiretroviral therapy. JPEN J Parenter Enteral Nutr 2018; 42: 380-386. 
21. Willig A, Wright L, Galvin TA. Practice paper of the Academy of Nutrition and Dietetics: nutrition intervention and human immunodeficiency virus infection. J Acad Nutr Diet 2018; 118: 486-498.

22. Polo R, Gómez-Candela C, Miralles C, et al. Recomendaciones de SPNS/GEAM/SENBA/SENPE/AEDN/SEDCA/GESIDA sobre nutrición en el paciente con infección por VIH. Nutr Hosp 2007; 22: 229-243.

23. Anastasi JK, Capili B, Kim AG, McMahon D, Heitkemper MM. Symptom management of HIV-related diarrhea by using normal foods: a randomized controlled clinical trial. J Assoc Nurses AIDS Care JANAC 2006; 17: 47-57.

24. Zahedi MJ, Behrouz V, Azimi M. Low fermentable oligo-di-monosaccharides and polyols diet versus general dietary advice in patients with diarrhea-predominant irritable bowel syndrome: a randomized controlled trial. J Gastroenterol Hepatol 2018; 33: 1192 1199.

25. Gecse K, Róka R, Séra T, et al. Leaky gut in patients with diarrhea-predominant irritable bowel syndrome and inactive ulcerative colitis. Digestion 2012; 85: 40-46.

26. Herzog K, Williams R, Cho I, Tenner C, Poles M. Irritable bowel syndrome and HIV: a cross sectional study of the severity of gastrointestinal symptoms and HIV-infected subjects: 1302. Am J Gastroenterol 2010; 105: S479.

27. Shariati A, Fallah F, Pormohammad A, et al. The possible role of bacteria, viruses, and parasites in initiation and exacerbation of irritable bowel syndrome. J Cell Physiol 2019; 234: 8550-8569.

28. Pedersen N, Ankersen DV, Felding M, et al. Low-FODMAP diet reduces irritable bowel symptoms in patients with inflammatory bowel disease. World J Gastroenterol 2017; 23: 3356-3366.

29. Vazquez-Roque MI, Camilleri M, Smyrk T, et al. A Controlled trial of gluten-free diet in patients with irritable bowel syndrome-diarrhea: effects on bowel frequency and intestinal function. Gastroenterology 2013; 144: 903-911.e3.

30. Alexander DD, Bylsma LC, Elkayam L, Nguyen DL. Nutritional and health benefits of semi-elemental diets: a comprehensive summary of the literature. World J Gastrointest Pharmacol Ther 2016; 7: 306-319.

31. Salomon SB, Jung J, Voss T, Suguitan A, Rowe WB, Madsen DC. An elemental diet containing medium-chain triglycerides and enzymatically hydrolyzed protein can improve gastrointestinal tolerance in people infected with HIV. J Am Diet Assoc 1998; 98: 460-462.

32. Craig GB, Darnell BE, Weinsier RL, et al. Decreased fat and nitrogen losses in patients with AIDS receiving medium-chain-triglyceride-enriched formula vs those receiving long-chain-triglyceride-containing formula. J Am Diet Assoc 1997; 97: 605-611.

33. Ockenga J, Grimble R, Jonkers-Schuitema C, et al. ESPEN Guidelines on enteral nutrition: wasting in HIV and other chronic infectious diseases. Clin Nutr 2006; 25: 319-329.

34. Edwards WM, Drexler AM, Aboulafia DM, McCormick WC. Efficacy of total parenteral nutrition in a series of end-stage AIDS patients: a case-control study. AIDS Patient Care STDs 1997; 11: 323-329.

35. Worthington P, Balint J, Bechtold M, et al. When is parenteral nutrition appropriate? JPEN J Parenter Enteral Nutr 2017; 41: 324-377.

36. WHO. Nutritional care and support for people living with HIV/ AIDS. WHO n.d. Available at: http://www.who.int/nutrition/publications/hivaids/9789241591898/en/ (Accessed: 3.06.2019).

37. Scrimgeour AG, Lukaski HC. Zinc and diarrheal disease: current status and future perspectives. Curr Opin Clin Nutr Metab Care 2008; 11: 711-717.

38. WHO. Zinc supplementation in the management of diarrhoea WHO n.d. Available at: http://www.who.int/elena/titles/bbc/zinc diarrhoea/en/ (Accessed: 30.10.2018)
39. Lazzerini M, Wanzira $\mathrm{H}$. Oral zinc for treating diarrhoea in children. Cochrane Database Syst Rev 2016; 12: CD005436.

40. Cárcamo C, Hooton T, Weiss NS, et al. Randomized controlled trial of zinc supplementation for persistent diarrhea in adults with HIV-1 infection. J Acquir Immune Defic Syndr 2006; 43: 197-201.

41. Baum MK, Lai S, Sales S, Page JB, Campa A. Randomized, controlled clinical trial of zinc supplementation to prevent immunological failure in HIV-infected adults. Clin Infect Dis 2010; 50: 1653-1660.

42. Zeng L, Zhang L. Efficacy and safety of zinc supplementation for adults, children and pregnant women with HIV infection: systematic review. Trop Med Int Health 2011; 16: 1474-1482.

43. Achamrah N, Déchelotte P, Coëffier M. Glutamine and the regulation of intestinal permeability: from bench to bedside. Curr Opin Clin Nutr Metab Care 2017; 20: 86-91.

44. Noyer CM, Simon D, Borczuk A, Brandt LJ, Lee MJ, Nehra V. A double-blind placebo-controlled pilot study of glutamine therapy for abnormal intestinal permeability in patients with AIDS. Am J Gastroenterol 1998; 93: 972-975.

45. Bushen OY, Davenport JA, Lima AB, et al. Diarrhea and reduced levels of antiretroviral drugs: improvement with glutamine or alanylglutamine in a randomized controlled trial in Northeast Brazil. Clin Infect Dis 2004; 38: 1764-1770.

46. Huffman FG, Walgren ME. L-glutamine supplementation improves nelfinavir-associated diarrhea in HIV-infected individuals. HIV Clin Trials 2003; 4: 324-329.

47. Leite RD, Lima NL, Leite CAC, Farhat CK, Guerrant RL, Lima AAM. Improvement of intestinal permeability with alanyl-glutamine in HIV patients: a randomized, double blinded, placebo-controlled clinical trial. Arq Gastroenterol 2013; 50: 56-63.

48. Bertrand J, Ghouzali I, Guérin C, et al. Glutamine restores tight junction protein claudin-1 expression in colonic mucosa of patients with diarrhea-predominant irritable bowel syndrome. JPEN J Parenter Enteral Nutr 2016; 40: 1170-1176.

49. Vanek VW, Matarese LE, Robinson M, et al. A.S.P.E.N. position paper: parenteral nutrition glutamine supplementation. Nutr Clin Pract 2011; 26: 479-494.

50. Generoso S de V, Lages PC, Correia MITD. Fiber, prebiotics, and diarrhea: what, why, when and how. Curr Opin Clin Nutr Metab Care 2016; 19: 388-393.

51.. McClave SA, Taylor BE, Martindale RG, et al. Guidelines for the provision and assessment of nutrition support therapy in the adult critically ill patient: Society of Critical Care Medicine (SCCM) and American Society for Parenteral and Enteral Nutrition (A.S.P.E.N.). JPEN J Parenter Enteral Nutr 2016; 40: 159-211.

52. McFarland LV, Goh S. Are probiotics and prebiotics effective in the prevention of travellers' diarrhea: a systematic review and meta-analysis. Travel Med Infect Dis 2019; 27: 11-19.

53. Sherman DS, Fish DN. Management of protease inhibitor-associated diarrhea. Clin Infect Dis 2000; 30: 908-914.

54. McRorie JW, McKeown NM. Understanding the physics of functional fibers in the gastrointestinal tract: an evidence-based approach to resolving enduring misconceptions about insoluble and soluble fiber. J Acad Nutr Diet 2017; 117: 251-264.

55. Schiller LR. Antidiarrheal Drug Therapy. Curr Gastroenterol Rep 2017; 19: 18

56. Lertpipopmetha K, Kongkamol C, Sripongpun P. Effect of psyllium fiber supplementation on diarrhea incidence in enteral tubefed patients: a prospective, randomized, and controlled trial. JPEN J Parenter Enteral Nutr 2019; 43: 759-767.

57. Hill C, Guarner F, Reid G, et al. Expert consensus document. The International Scientific Association for Probiotics and Prebiotics consensus statement on the scope and appropriate use of the term probiotic. Nat Rev Gastroenterol Hepatol 2014; 11: 506-514.

58. Allen SJ, Martinez EG, Gregorio GV, Dans LF. Probiotics for treating acute infectious diarrhoea. Cochrane Database Syst Rev 2010; 11: CD003048. 
59. Bernaola Aponte G, Bada Mancilla CA, Carreazo Pariasca NY, Rojas Galarza RA. Probiotics for treating persistent diarrhoea in children. Cochrane Database Syst Rev 2010; 11: CD007401.

60. D’Angelo C, Reale M, Costantini E, D’Angelo C, Reale M, Costantini E. Microbiota and probiotics in health and HIV Infection. Nutrients 2017; 9: 615.

61. Carter GM, Esmaeili A, Shah H, et al. Probiotics in human immunodeficiency virus infection: a systematic review and evidence synthesis of benefits and risks. Open Forum Infect Dis 2016; 3: ofw164.

62. Santos ASEA de C, Silveira EA da, Falco MO, Nery MW, Turchi MD. Effectiveness of nutritional treatment and synbiotic use on gastrointestinal symptoms reduction in HIV-infected patients: Randomized clinical trial. Clin Nutr 2017; 36: 680-685.

63. Wilson NL, Moneyham LD, Alexandrov AW. A systematic review of probiotics as a potential intervention to restore gut health in HIV infection. J Assoc Nurses AIDS Care 2013; 24: 98-111.

64. Stadlbauer V. Immunosuppression and probiotics: are they effective and safe? Benef Microbes 2015; 6: 823-828. 